\title{
Disease, perceived infectibility and threat reactivity: a COVID-19 study
}

\section{Safra, L. ${ }^{1}$, Sijilmassi, A. ${ }^{2}$, Chevallier, C. ${ }^{3}$}

${ }^{1}$ Sciences Po, CEVIPOF, CNRS, Paris, France

${ }^{2}$ Institut Jean Nicod, Département d'études cognitives, ENS, EHESS, PSL Research University, CNRS, Paris France

${ }^{3}$ Laboratoire de Neurosciences Cognitives, Département d'études cognitives, ENS, PSL, Research University, INSERM, Paris France

\section{Pre-registration statement}

We applied the same analyses as those pre-registered following the collection of the first wave (https://osf.io/5cexn/). The results presented in the paper were conducted on the final sample, in other words on the participants who completed both waves. In addition, after the pre-registration, we automatically coded the correspondence between postal codes and upper tier local areas, which corrected issues due to the initial manual coding.

\section{Declaration of interest}

None.

\section{Funding}

This study was supported by the Institut d'Études Cognitives (ANR-17-EURE-0017 FrontCog and ANR-10-IDEX-0001-02 PSL). 


\section{Abstract}

8

9 Using a two-wave online experiment, we investigate whether COVID-19 exposure changes participants' threat-detection threshold. Threat reactivity was measured in a

11 signal detection task among 277 British adults who also reported how vulnerable they 12 felt to infectious diseases. Participants' data were then matched to the local number of 13 confirmed COVID-19 cases announced by the NHS every day. We found that participants 14 who perceive themselves as more likely to catch infectious diseases displayed higher 15 threat reactivity in response to increased COVID-19 cases.

\section{Keywords}

21 Threat reactivity; disease vulnerability; pathogen threat; COVID-19; error-management 22 theory 
Having an accurate perception of potential risks and benefits is essential to optimal decision-making. Although all errors should ideally be avoided, some errors are costlier than others. False positives and false negatives indeed have asymmetric consequences for the individual because failing to detect a threat when there is one is more dangerous than believing that there is a threat when there is none (Haselton \& Buss, 2000). Given this asymmetry, error-management theory predicts that individuals should be biased to overdetect negative events. In line with this idea, surveys consistently show that people overperceive all kinds of threats, such as crime rates, terrorism or unemployment risk (Gilbert et al., 2016; Lieder et al., 2016).

To be adaptive however, threat reactivity must be sensitive to individual circumstances, such as physical condition or age (Nettle \& Bateson, 2012). For example, people in poor physical condition can escape less easily from threats, which means that for them, more so than for people in better shape, having a lower threat detection threshold is particularly adaptive. In line with this idea, people in poor physical shape are more prone to the auditory looming effect, a bias that helps us get ready to move away from approaching objects by making us hear sounds that are coming towards us as closer than sounds going away from us (Neuhoff et al., 2012). Similarly, individuals who perceive themselves as more sensitive to diseases have stronger reactions to disease cues (Navarrete \& Fessler, 2006; Reid et al., 2012); and women who perceive themselves as more vulnerable to sexual coercion tend to stereotype out-group males as more threatening (McDonald et al., 2015) 
In addition to these internal factors, readiness to react to threat should also respond to local circumstances. For instance, in a highly threatening environment, individuals should react more readily to threat cues than in an environment with fewer threats (Nettle \& Bateson, 2012). In line with this idea, social perception experiments have shown that maltreated children are more likely to over-detect anger in emotionally ambiguous faces

54 (Pollak, 2008).

55

In the present paper, we further test this model by analyzing the effect of the COVID-19 epidemic on individuals' threat reactivity. Using signal detection theory, we test the hypothesis that being surrounded by more COVID-19 cases, as measured by local COVID19 prevalence, will increase people's reactivity to threats. More specifically, we predict that this effect is modulated by individuals' perceived vulnerability to infectious diseases, with a larger effect among individuals who perceive themselves as highly vulnerable to diseases.

63

In order to test this hypothesis, we assessed subjective vulnerability in a sample of participants who took part in a threat reactivity experiment twice: on March 24th and two weeks later, on April $7^{\text {th }}$. Local variations of the number of COVID-19 cases allow us to obtain quasi-experimental natural variations of threat exposure. For instance, on March $24^{\text {th }}$, the urban local tier of Wakefield reported only 13 cases, less than the mostly rural area of Wiltshire, which counted almost 3 times more cases. The evolution of the number of COVID-19 cases also varied from one region to the next. For instance, both Hounslow and Essex districts counted around 80 cases on March 24th but Essex district counted 938 cases two weeks later while Hounslow district counted only 279 more cases at that date. 
Similarly, while the district of Merton ranked $21^{\text {st }}$ in the number of cases on Match $24^{\text {th }}$ it fell to the $40^{\text {th }}$ position on April $7^{\text {th }}$. Conversely, the district of Liverpool jumped from the $4^{\text {th }}$ to $17^{\text {th }}$ position in that same time interval. We thus exploited these local variations in order to assess the combined effect of exposure to disease threat and subjective vulnerability to diseases on participants' reactivity to threat.

\section{Methods}

Ethics Statement. Our study was approved by the local Ethical Committee (CERES $\mathrm{n}^{\circ} 201659$ ). Each participant received a description of the study and provided their informed consent before starting the experiment. All scripts and raw data are available in the OSF project (https://osf.io/5cexn/).

Participants. 352 UK participants recruited on Prolific Academic completed our study twice: once between March 24th at $2 \mathrm{PM}$ and March $25^{\text {th }}$ at $2 \mathrm{PM}$, and a second time between April $7^{\text {th }}$ at $2 \mathrm{PM}$ and April $8^{\text {th }}$ at $2 \mathrm{PM}$. The $2 \mathrm{PM}$ limit was chosen because daily figures of COVID19 cases are announced by the British government at that time. All participants received compensation for their time ( $€ 5$ per hour), as well as a variable bonus depending on task performance ( $£ 0-0.50)$. For both waves, all trials with reaction times below $150 \mathrm{~ms}$ or above 2,500 ms were excluded. 1 participant was removed for having mean reaction times at \pm 3 SD of the mean in the first wave and 4 were excluded in the second wave. 22 participants were then removed for having reaction times outside these ranges on more than $40 \%$ of the trials in the first wave and 8 were excluded based on this same criterion in the second wave. In addition, 7 participants were removed for 

reached.

not having provided answers to the "Perceived Infectability and Germ Aversion" questionnaire in the first wave and 27 participants were removed for not having provided answers to the "Perceived Infectability and Germ Aversion" questionnaire in the second wave (Duncan et al., 2009). Finally, 4 participants were excluded for not having provided their date of birth and 2 participants were excluded from not having provided a valid postal code. In total, 75 participants were excluded, which left us with a final sample of 277 participants living in 112 geographical areas (158 Females, mean age: $38.43 \pm 12.58$ s.d. years).

Materials. The task was presented using Qualtrics. Monetary punishments were presented using text ("- 5 pennies"), which represented the true amount that was subtracted from participants' total bonus payment at the end of the experiment. The punishments were provided in response to incorrect identifications of a line appearing in the center of a circle as being short $(11.5 \mathrm{~mm})$ or long $(13 \mathrm{~mm})$. Following the experimental task, participants completed questionnaires including standard sociodemographic questions (age, income, education, postal code of residence) as well as their perceived sensitivity to diseases.

Design and Procedure. The experiment was conducted online and lasted approximately 25 minutes. Participants were told that their task was to classify a line as either short or long by pressing the corresponding key and that feedback for incorrect responses would occur some of the time. The training phase consisted of 22 practice trials during which the difficulty of the task was progressively raised until real-game conditions were 
The experiment consisted of 300 trials separated in three 100-trial blocks. Each trial began with the presentation of a fixation cross $(300 \mathrm{~ms})$, followed by an empty circle (300ms). The short or the long line was then flashed within the circle (40ms) and disappeared to show a plain black screen during which participants could submit their response (Figure 1). An equal number of short and long lines were presented within each block. Short and long lines were presented in a random order. Participants were given an infinite amount of time to indicate their response using 'e' or ' $p$ ' on the keyboard. Based on the reinforcing schedule, incorrect responses were followed by an $800 \mathrm{~ms}$ negative feedback screen with a probability of $75 \%$ or $25 \%$, depending on the type of stimulus (see below). Otherwise, the next trial was immediately presented.

An asymmetrical reinforcement ratio was introduced such that one type of line (i.e. short or long) was punished more often. The line type that was punished more frequently is referred to as the "harsh stimulus" and the line type that was punished less frequently is referred to as the "lenient stimulus". Misidentifying the harsh line (i.e., if the harsh line is short, a misidentification is to respond "long") was followed by a negative feedback with a probability of $75 \%$. Misidentifying the lenient line (i.e., if the lenient line is long, a misidentification is to respond "short") was followed by a negative feedback with a probability of $25 \%$. Analyses from a pilot study using the same behavioral task prove that the actual distribution of negative feedbacks corresponds to the anticipated asymmetric ratio design: on average, $76.1 \%$ of errors on the Harsh line and $29.4 \%$ of errors on the Lenient line were followed by a negative feedback ( -5 pennies). These results are consistent across conditions and blocks. $74 \%$ of errors on harsh lines and 28\% of errors on lenient lines were punished in Block 1; 74\% and 30\% in Block 2; and 74\% and 21\% in Block 3 (Safra et al., 2021). 
149 The long line was randomly assigned to being the harsh or the lenient stimulus for each participant.

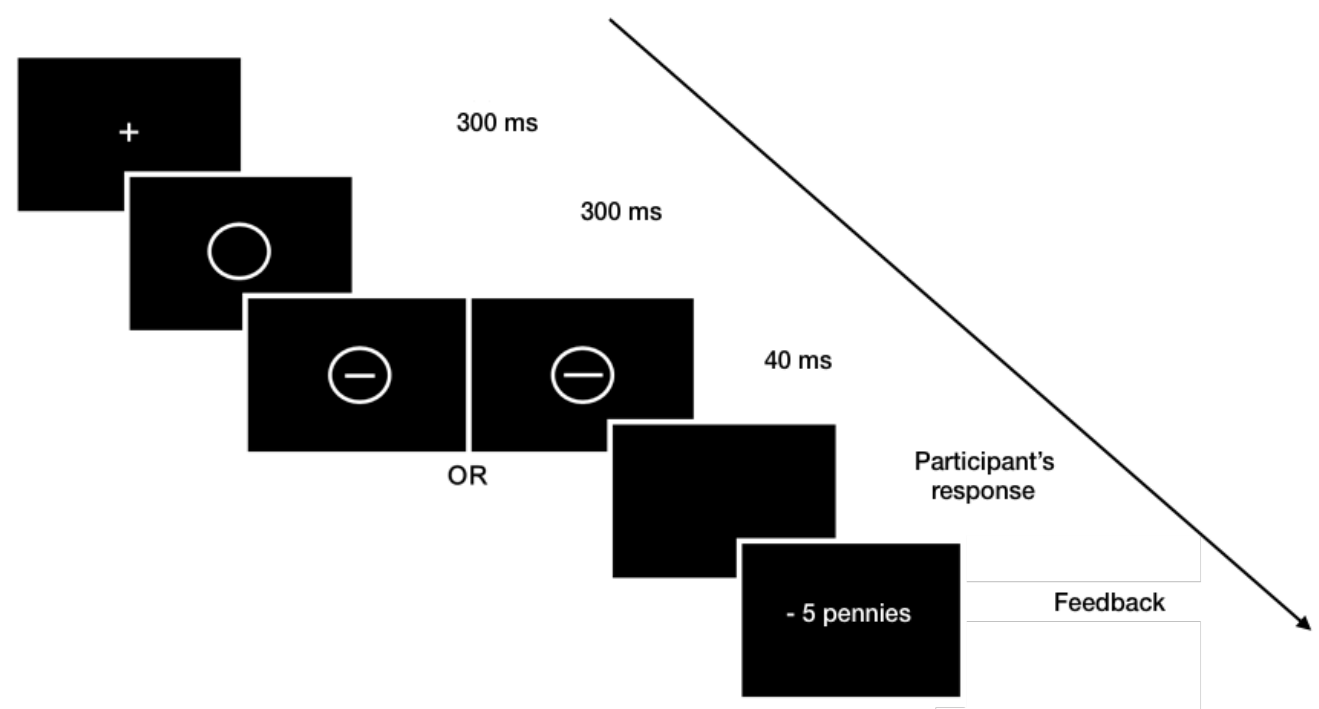

151

Figure 1. Schematic representation of the tasks A fixation cross appears for $300 \mathrm{~ms}$, followed by an empty circle. A short or a long line is then flashed inside the circle for $40 \mathrm{~ms}$. Participants have an infinite amount of time to respond before they receive a punishment for some of their incorrect responses.

Threat reactivity. Threat reactivity was conceptualized as participants' bias to avoid punishment. In our task this meant over-detecting the line for which misidentification was associated with more frequent punishment (i.e., the harsh line). Response bias towards the harsh line was computed using the standard signal detection measure:

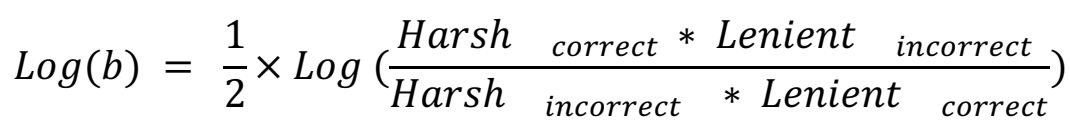

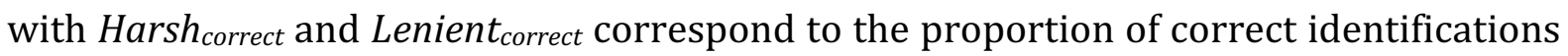
(hits and correct rejections) to the total number of harsh and lenient trials respectively, 
and Harsh incorrect $_{\text {and Lenient }}$ incorrect correspond to the proportion of false identifications

167 (misses and incorrect rejections) to the total number of harsh and lenient trials

168 respectively. When accuracy was equal to 1 or 0 , we followed the log linear correction

169 procedure described by Hautus (1995).

170

171 Following Pizzagalli et al. (2005) and Chevallier et al. (2016), reactivity to threat was computed by measuring the change in bias towards the harsh line between the first block (in which the participant is naive about the value of the two lines) and the last block (in which the participant has experienced that the misidentification of one line is more frequently associated with punishments).

Perceived infectability. At the end of the experiment, participants' susceptibility to infectious diseases was assessed using the "Perceived Vulnerability to Disease" questionnaire (Duncan, Schaller \& Park, 2009). This questionnaire is composed of two subscales: the "Perceived Infectability" subscale which measures the self-reported susceptibility to infectious diseases, and the "Germ Aversion" subscale which assess the level of affective responses to situations of high risk of disease transmission. In our study, we used "Perceived Infectability" as our measure of interest as it indicates how much the individual feels threatened by infectious diseases, while "Germ Aversion" was used as a control measure for general affective reactions to diseases.

Participants completed these scales twice, once in Wave 1 and a second time in Wave 2. Our data shows strong consistency in participants' response to the perceived vulnerability to disease questionnaire. We found a strong inter-temporal correlation between the two waves for the global disgust score $(r=0.86 \pm 0.01$ s.e.m $\quad, p<.001)$, 
190

191

192

193

194

195

196

197

198

199

200

201

202

203

204

205

206

207

208

209

210

211

212

213

the perceived infectability score $(r=0.86 \pm 0.01$ s.e.m., $p<.001)$ and the germ aversion score $(r=0.84 \pm 0.02$ s.e.m, $p<.001)$.

Socio-demographic information. In Wave 1, participants provided information about their age, gender and level of education. Additional questionnaires were also included at the end of Wave 1 and Wave 2 (see Supplementary information for details).

Local COVID-19 exposure. The number of local COVID-19 cases was retrieved from the NHS website based on participants' postal codes. For residents of England, we retrieved COVID-19 cases statistics at the upper tier local authority level (UTLA) from the NHS website. UTLAs are an administrative subdivision below the level of the region. For residents of Scotland, COVID-19 figures were given at the level of health board areas from the Scotland NHS website (there are 14 such areas). Finally, COVID-19 cases statistics for Wales and Northern Ireland were not available for smaller subdivisions. We thus considered Wales and Northern Ireland as just other administrative sub-divisions.

Importantly, perceived vulnerability to pathogens was not significantly correlated with the local numbers of COVID-19 cases neither in Wave $1(r=.01 \pm 0.06$ s.e.m., $t(275)=$ $0.13, p>.250)$ nor in Wave $2(r=-.01 \pm 0.06$ s.e.m., $t(275)=-0.21, p>.250)$. Similarly, in both Waves, no significant correlation was found between the local number of COVID-19 cases and the subscales of Perceived Vulnerability to Disease: perceived infectability (Wave 1: $r=-.02 \pm 0.06$ s.e.m., $t(275)=-0.37, p>.250$; Wave $2: r=-.04 \pm 0.06$ s.e.m., $t(275)$ $=-0.59, p>.250$ ) and germ aversion (Wave 1: $r=.03 \pm 0.06$ s.e.m., $t(275)=0.52, p>.250$; Wave $2: r=.02 \pm 0.06$ s.e.m., $t(275)=0.26, p>.250)$.

\section{Analyses.}


214 Threat reactivity in Wave 1 and in Wave 2 were first analyzed using robust mixed linear

215 regressions taking the number of reported COVID-19 cases in the upper tier local 216 authority, germ aversion and perceived infectability, as well as the interaction between

217 local exposure to COVID-19 and these two dimensions of perceived vulnerability to 218 diseases as predictors. All the predictors were transformed into z-scores to avoid issues 219 due to scaling differences. To control for individual and local effects independent of the exposure to COVID-19 (such as living in a rural area or in a city), participant ID and the upper tier local authority was included as a random factor. In order to further assess the robustness of our results, we conducted additional models with age, gender and level of education as additional predictors.

224

Finally, in order to compare participants' behavior across the two waves, we analysed

Waves 1 and 2 simultaneously using similar robust mixed linear regressions as 227 previously but taking "Wave" as additional predictor. All the robust mixed linear regressions were conducted using robustlmm R package (Koller, 2016).

\section{Results}

No main effect of COVID-19 exposure was found on threat reactivity $(b=-0.07 \pm 0.06$ s.e.m., $z=-1.24, p=.215$ ). However, there was a significant positive effect of the interaction between exposure to COVID-19 and perceived infectability $(b=0.15, \pm 0.07$ s.e.m., $z=2.27, p=.023$ ) such that participants who perceived themselves as more likely to catch infectious diseases reacted more strongly to the number of COVID-19 cases in their surroundings (Table 1). This effect was robust to the inclusion of age, gender and level of education (interaction between perceived infectability and COVID-19 exposure: $b=0.18 \pm 0.07$ s.e.m., $z=2.48, p=.013$; no other significant effect, see Table 1 ) and was 
specific to perceived infectability as there was no significant effect of germ aversion

240 (main effect: $b=0.08 \pm 0.06$ s.e.m., $z=1.31, p=.190$; interaction: $b=-0.00 \pm 0.05$ s.e.m., $241 z=-0.01, p>.250)$.

242 Analysis of Waves 1 and 2 confirmed the positive interaction between exposure to COVID-

24319 and perceived infectability ( $b=0.10 \pm 0.05$ s.e.m., $z=2.22, p=.026$; Table 1$)$. This

244 effect was still found as a trend after including demographic variables as predictors $(b=$ $0.10 \pm 0.05$ s.e.m., $z=1.85, p=.064$; Table 1 ).

To sum up, differences in the number of COVID-19 cases had no overall effect on participants' threat reactivity but participants with a high sensitivity to diseases had a lower threat reactivity threshold when they lived in an area with many COVID-19 cases than when those who lived in an area with few COVID-19 cases. This suggests that threat reactivity increased in response to higher levels of disease threat among those who perceived themselves as vulnerable to diseases.

One possible interpretation of our findings is that participants with a higher perceived sensitivity to diseases are also those who are more informed. We ruled out this possibility by measuring participants' accuracy when estimating the number of COVID-19 cases in the UK. We found that perceived infectability was not correlated with participants' accuracy when they estimated the number of COVID-19 cases in the UK $(r=0.04 \pm 0.06$ s.e.m., $t(275)=0.66, p>.250$; non-signed error: $r=-0.04, t(275)=-0.63 \pm 0.06$ s.e.m., , $p>.250$; measured in Wave 2) and that the interaction between the number of COVID19 cases and perceived infectability remained significant after adjusting for participants' knowledge of the epidemics (after controlling for signed error: $b=0.09 \pm 0.05$ s.e.m., $z=$ 1.97, $p=.049$; after controlling for non-signed error: $b=0.09 \pm 0.05$ s.e.m., $z=1.89, p=$ .058). This suggests that participants with a high level of perceived infectability do not 
263 have a better or a more catastrophic image of the situation than those with a low level of 264 perceived infectability. 


\begin{tabular}{|c|c|c|c|c|}
\hline & \multicolumn{2}{|c|}{ Wave 1} & \multicolumn{2}{|c|}{ Waves $1 \& 2$} \\
\hline & Reduced model & Full model & Reduced model & Full model \\
\hline Intercept & $\begin{array}{l}0.23 \pm 0.06^{* * *} \\
z=4.09\end{array}$ & $\begin{array}{l}0.28 \pm 0.08^{* * *} \\
z=3.70\end{array}$ & $\begin{array}{l}0.21 \pm 0.06^{* * *} \\
z=3.49\end{array}$ & $\begin{array}{l}0.27 \pm 0.07^{* * *} \\
z=3.77\end{array}$ \\
\hline $\begin{array}{l}\text { COVID-19 } \\
\text { published cases }\end{array}$ & $\begin{array}{l}-0.07 \pm 0.06 \\
z=-1.24\end{array}$ & $\begin{array}{l}-0.09 \pm 0.09 \\
z=-0.96\end{array}$ & $\begin{array}{l}-0.05 \pm 0.05 \\
z=-1.05\end{array}$ & $\begin{array}{l}-0.04 \pm 0.07 \\
z=-0.54\end{array}$ \\
\hline \multirow{4}{*}{$\begin{array}{l}\text { Perceived } \\
\text { infectability }\end{array}$} & \multicolumn{4}{|c|}{ Main effect } \\
\hline & $\begin{array}{l}-0.09 \pm 0.06 \\
z=-1.63\end{array}$ & $\begin{array}{l}-0.10 \pm 0.06 \\
z=-1.66\end{array}$ & $\begin{array}{l}-0.05 \pm 0.04 \\
z=-1.25\end{array}$ & $\begin{array}{l}-0.06 \pm 0.04 \\
z=-1.53\end{array}$ \\
\hline & \multicolumn{4}{|c|}{ Interaction with COVID-19 published cases } \\
\hline & $\begin{array}{l}0.15 \pm 0.07 * \\
z=2.27\end{array}$ & $\begin{array}{l}0.18 \pm 0.07 * \\
z=2.48\end{array}$ & $\begin{array}{l}0.10 \pm 0.05 * \\
z=2.22\end{array}$ & $\begin{array}{l}0.10 \pm 0.06^{\circ} \\
z=1.85\end{array}$ \\
\hline \multirow{4}{*}{ Germ aversion } & \multicolumn{4}{|c|}{ Main effect } \\
\hline & $\begin{array}{l}0.08 \pm 0.06 \\
Z=1.48\end{array}$ & $\begin{array}{l}0.08 \pm 0.06 \\
Z=1.31\end{array}$ & $\begin{array}{l}0.06 \pm 0.04 \\
Z=1.48\end{array}$ & $\begin{array}{l}0.06 \pm 0.04 \\
Z=1.47\end{array}$ \\
\hline & \multicolumn{4}{|c|}{ Interaction with COVID-19 published cases } \\
\hline & $\begin{array}{l}1.01 \pm 0.05 \\
z=0.17\end{array}$ & $\begin{array}{l}-0.00 \pm 0.05 \\
z=-0.01\end{array}$ & $\begin{array}{l}-0.04 \pm 0.03 \\
z=-1.30\end{array}$ & $\begin{array}{l}-0.05 \pm 0.03 \\
z=-1.39\end{array}$ \\
\hline \multirow{4}{*}{ Age } & \multicolumn{4}{|c|}{ Main effect } \\
\hline & & $\begin{array}{l}-0.02 \pm 0.06 \\
z=-0.36\end{array}$ & & $\begin{array}{l}0 . \quad 04 \pm 0.04 \\
z=1.10\end{array}$ \\
\hline & \multicolumn{4}{|c|}{ Interaction with the number of COVID-19 published cases } \\
\hline & & $\begin{array}{l}-0.05 \pm 0.08 \\
z=-0.63\end{array}$ & & $\begin{array}{l}-0.04 \pm 0.05 \\
z=-0.72\end{array}$ \\
\hline \multirow{4}{*}{ Gender } & \multicolumn{4}{|c|}{ Main effect } \\
\hline & & $\begin{array}{l}-0.12 \pm 0.12 \\
z=-1.06\end{array}$ & & $\begin{array}{l}-0.12 \pm 0.08 \\
z=-1.49\end{array}$ \\
\hline & Inter & cion with the nur & ff COVID-19 publi & d cases \\
\hline & & $\begin{array}{l}0.05 \pm 0.12 \\
z=0.43\end{array}$ & & $\begin{array}{l}-0.00 \pm 0.09 \\
z=-0.02\end{array}$ \\
\hline \multirow{4}{*}{ Education level } & \multicolumn{4}{|c|}{ Main effect } \\
\hline & & $\begin{array}{l}1.01 \pm 0.06 \\
z=0.18\end{array}$ & & $\begin{array}{l}-0.01 \pm 0.04 \\
z=-0.15\end{array}$ \\
\hline & \multicolumn{4}{|c|}{ Interaction with the number of COVID-19 published cases } \\
\hline & & $\begin{array}{l}-0.07 \pm 0.08 \\
z=-0.86\end{array}$ & & $\begin{array}{l}-0.04 \pm 0.05 \\
z=-0.72\end{array}$ \\
\hline Wave & & & $\begin{array}{l}-0.01 \pm 0.09 \\
z=-0.09\end{array}$ & $\begin{array}{l}-0 . \quad 03 \pm 0.09 \\
z=-0.36\end{array}$ \\
\hline
\end{tabular}

Table 1. Coefficients of the mixed linear regression on threat reactivity conducted on Wave 1 alone and Waves

1 and 2 simultaneously. A positive interaction was found between perceived infectability and the number of COVID-

19 published cases in the local area. Standardized regression coefficients are presented with the standard error to mean and the associated $\mathrm{z}$ value. ${ }^{\circ}$ indicates a p-value inferior to $.100, *$ indicates a p-value inferior to .050 and ${ }^{* * *}$ indicates a p-value inferior to .001. 
273 In line with our hypothesis, our results revealed that variations in threat reactivity was associated to a combination of local threat level (as measured by the local prevalence of COVID-19) and individual sensitivity to threat: the more individuals perceived themselves as susceptible to infectious diseases, the more COVID-19 exposure increased their reactivity to threats. This effect was present at the two time-points of the epidemics we had access to: on the day following the official lockdown enforcement in the UK and two weeks later. These results provide evidence that individuals react to the presence of survival threats by decreasing their threshold for responding to punishment (Nettle \& Bateson, 2012). These results are in line with previous findings by Makhanova \& Shepherd (2020) suggesting that perceived infectability to diseases is associated with increased vigilance to health and disease-related issues in the context of COVID-19 pandemic.

Going further, the present study reveals that psychological adjustments following an increase of threat in the environment acts at the global level of individuals' psychology. More precisely, it appears that disease threat, such as COVID-19 exposure, modulates threat reactivity across domains, even if the threat is not linked to health, as it is the case with the financial punishments used in our experiment. exposure suggests that this response is conditional on the level of perceived threat and not on the absolute level of threat in the environment. Therefore, it appears that this effect is genuinely mediated by individuals' perceived risks and benefits of adopting 
different strategies in the response to an environmental change. Importantly, this effect was independent from individuals' knowledge of the epidemic as well as their age, gender and level of education.

In a nutshell, our study provides evidence that individuals react to the presence of threats in their environment by decreasing their threshold for reacting to threats. Moreover, our results show that adaptation of low-level psychological variables is conditional on how much the threat that is present in the environment corresponds to a threat subjectively perceived as such by the individual. This underlines the importance of taking interindividual differences into account when designing public policies in response to large scale public threats.

\section{References}

Chevallier, C., Tonge, N., Safra, L., Kahn, D., Kohls, G., Miller, J., \& Schultz, R. T. (2016). Measuring Social Motivation Using Signal Detection and Reward Responsiveness. PLOS ONE, 11(12), e0167024. https://doi.org/10.1371/journal.pone.0167024

Duncan, L. A., Schaller, M., \& Park, J. H. (2009). Perceived vulnerability to disease : Development and validation of a 15-item self-report instrument. Personality and Individual

Differences, $47(6)$ $541-546$. https://doi.org/10.1016/j.paid.2009.05.001

Gilbert, J., Uggla, C., \& Mace, R. (2016). Knowing your neighbourhood : Local ecology and personal experience predict neighbourhood perceptions in Belfast, Northern Ireland. Royal Society Open Science, 3(12), 160468. https://doi.org/10.1098/rsos.160468 
Haselton, M. G., \& Buss, D. M. (2000). Error management theory : A new perspective on biases in cross-sex mind reading. Journal of Personality and Social Psychology, 78(1), 81-91. https://doi.org/10.1037//0022-3514.78.1.81

Hautus, M. J. (1995). Corrections for extreme proportions and their biasing effects on estimated values ofd'. Behavior Research Methods, Instruments, \& Computers, 27(1), 46-51. https://doi.org/10.3758/BF03203619

Koller, M. (2016). robustlmm : An R Package for Robust Estimation of Linear MixedEffects Models. Journal of Statistical Software, 75(6), 1-24. https://doi.org/10.18637/jss.v075.106

Lieder, F., Griffiths, T., \& Hsu, M. (2016). Over-representation of extreme events in decision-making reflects rational use of cognitive resources. In OSF Preprints (kxxag; OSF Preprints). Center for Open Science. https://ideas.repec.org/p/osf/osfxxx/kxxag.html

Makhanova, A., \& Shepherd, M. A. (2020). Behavioral immune system linked to responses to the threat of COVID-19. Personality and Individual Differences, 167, 110221. https://doi.org/10.1016/j.paid.2020.110221

McDonald, M. M., Donnellan, M. B., Cesario, J., \& Navarrete, C. D. (2015). Mate choice preferences in an intergroup context: Evidence for a sexual coercion threatmanagement system among women. Evolution and Human Behavior, 36(6), 438-445. https://doi.org/10.1016/j.evolhumbehav.2015.04.002

Navarrete, C. D., \& Fessler, D. M. T. (2006). Disease avoidance and ethnocentrism: The effects of disease vulnerability and disgust sensitivity on intergroup attitudes. Evolution and Human Behavior, 27(4), 270-282. https://doi.org/10.1016/j.evolhumbehav.2005.12.001 
Nettle, D., \& Bateson, M. (2012). The Evolutionary Origins of Mood and Its Disorders. Current Biology, 22(17), R712-R721. https://doi.org/10.1016/j.cub.2012.06.020

Neuhoff, John G., Katherine L. Long, and Rebecca C. Worthington. "Strength and physical fitness predict the perception of looming sounds." Evolution and Human Behavior 33.4 (2012): 318-322.

Pizzagalli, D. A., Jahn, A. L., \& O’Shea, J. P. (2005). Toward an objective characterization of an anhedonic phenotype: A signal-detection approach. Biological Psychiatry, 57(4), 319-327. https://doi.org/10.1016/j.biopsych.2004.11.026

Reid, S. A., Zhang, J., Anderson, G. L., Gasiorek, J., Bonilla, D., \& Peinado, S. (2012). Parasite primes make foreign-accented English sound more distant to people who are disgusted by pathogens (but not by sex or morality). Evolution and Human Behavior, 33(5), 471-478. https://doi.org/10.1016/j.evolhumbehav.2011.12.009

Safra, L., Chevallier, C., \& Sijilmassi, A. (2021). Poverty and Threat Reactivity. https://doi.org/10.31234/osf.io/fp35r 